\title{
Impaired decision making and feedback evaluation in borderline personality disorder
}

\author{
B. Schuermann ${ }^{1 *}$, N. Kathmann ${ }^{1}$, C. Stiglmayr ${ }^{1}$, B. Renneberg ${ }^{2}$ and T. Endrass ${ }^{1}$ \\ ${ }^{1}$ Humboldt-Universität zu Berlin, Berlin, Germany \\ ${ }^{2}$ Freie Universität Berlin, Berlin, Germany
}

Background. Increased impulsivity is considered to be a core characteristic of borderline personality disorder (BPD) and has been shown to play a significant role in decision making and planning. Neuropsychological studies in BPD revealed impairments of executive functions, and it is assumed that these deficits are related to altered feedback processing. However, research on executive functions in BPD is still limited and the underlying deficits remain an open question. The present study, therefore, explored whether decision-making deficits are related to altered feedback evaluation in BPD.

Method. A total of 18 BPD patients and 18 matched healthy controls underwent a modified version of the Iowa Gambling Task while an electroencephalogram was recorded. Feedback processing was examined by measuring the feedback-related negativity (FRN) and the P300 as electrophysiological correlates of feedback evaluation.

Results. Behavioural results revealed that BPD patients, relative to controls, made more risky choices and did not improve their performance. With regard to the FRN, amplitudes in BPD patients did not discriminate between positive and negative feedback information. Further, BPD patients showed reduced FRN amplitudes, which were associated with enhanced impulsivity and enhanced risk taking. In contrast, the P300 amplitudes following negative feedback were increased in BPD patients, relative to controls.

Conclusions. This study indicates that BPD patients are impaired in decision making, which might be related to a dysfunctional use of feedback information. Specifically, BPD patients did not learn to avoid disadvantageous selections, even though they attended to negative consequences.

Received 13 July 2010; Revised 10 December 2010; Accepted 15 December 2010; First published online 24 January 2011

Key words: Borderline personality disorder, decision making, event-related brain potentials, feedback evaluation.

\section{Introduction}

Borderline personality disorder (BPD) is a mental disorder characterized by increased impulsivity and, as a consequence, by increased risk-taking behaviour (Links et al. 1999; APA, 2000). Executive dysfunctions are assumed to underlie the phenotypic features of BPD, especially increased impulsivity (Bazanis et al. 2002; Lenzenweger et al. 2004). Neuropsychological studies in BPD suggest impairments in multiple cognitive domains, with decision making or planning most frequently affected (Ruocco, 2005; LeGris \& van Reekum, 2006). Neuroimaging studies also support the notion that brain regions involved in impulse control and decision making are altered in BPD. Impairments include volume loss and hypometabolism

* Address for correspondence: Dipl.-Psych. B. Schuermann, Humboldt-Universität zu Berlin, Institut für Psychologie, Rudower Chaussee 18, 12489 Berlin, Germany.

(Email : beate.schuermann@hu-berlin.de) of the orbitofrontal cortex (OFC) and the anterior cingulate cortex (ACC; De La Fuente et al. 1997; Tebartz van Elst et al. 2003). OFC lesions have been associated with reduced performance in reinforcement learning because of a potential inability to modify behaviour in response to feedback (Rolls et al. 1994; Berlin et al. 2005). The ACC plays a pivotal role in the detection and evaluation of unfavourable outcomes (Ridderinkhof et al. 2004). It is highly associated with risk prediction, including signalling the extent of risk and the severity of consequences (Brown \& Braver, 2007).

Decision making and impulsivity have been repeatedly examined with the Iowa Gambling Task (IGT; Bechara et al. 1994). The IGT is a complex paradigm that is supposed to reflect real-life decision making in the way it considers uncertainty, rewards and punishment. It allows the investigation of persistent learning difficulties, and it has been suggested that deficits reflect a reduced ability to avoid negative feedback information (Bechara et al. 2000). Recent 
studies using the IGT showed that BPD patients, relative to healthy controls, made fewer advantageous and goal-directed decisions and exhibited reduced learning (Haaland \& Landro, 2007; Maurex et al. 2009). Specifically, decision-making deficits have been interpreted as a deficit to use feedback information from previous trials to make current decisions (Bechara et al. 2000). To our knowledge, alterations in feedback evaluation have not been examined yet in BPD. Therefore, the main objective of the present study was to investigate feedback processing in BPD to further understand decision-making dysfunctions in these patients. Event-related brain potentials (ERPs) are used to elucidate the relationship between neural responses to feedback and decision making. Previous research on performance monitoring has identified negative-going ERP components that occur shortly after incorrect responses or after negative performance feedback. The error-related negativity (ERN; Falkenstein et al. 1990; Gehring et al. 1990) arises following the execution of erroneous responses and the feedback-related negativity (FRN; Miltner et al. 1997) is elicited by negative performance feedback when outcomes are worse than expected (Holroyd et al. 2002). Frank et al. (2005) showed that the FRN magnitude predicts the degree to which participants learn about the negative consequences of their decisions. ERN and FRN are both assumed to originate in the ACC (Gehring \& Willoughby, 2002; Debener et al. 2005) and to reflect neural processes in reinforcement learning and behavioural adjustment (Holroyd \& Coles, 2002).

Decision making and feedback evaluation have also been linked to the feedback-related P300. This ERP component is suggested to reflect the activity of a noradrenergic system associated with motivational processes (Nieuwenhuis et al. 2005). In gambling tasks, such as the IGT, the P300 varied with outcome magnitude, regardless of whether the outcome is a gain or a loss (Sato et al. 2005; Polezzi et al. 2009). Further, its amplitude is modulated by expectations, with enhanced amplitudes to unexpected feedback than to expected feedback (Hajcak et al. 2005, 2007). In sum, the P300 amplitude might index feedback salience (De Bruijn et al. 2004; Yeung \& Sanfey, 2004) and thus is associated with the motivational significance of feedback. In contrast, the FRN reflects whether the feedback is consistent with expectations and is associated with the efficacy of learning.

Individual differences in impulsivity and risktaking behaviour have been linked to modulations in ERN amplitudes. Highly impulsive individuals and BPD patients exhibit smaller ERN amplitudes (De Bruijn et al. 2006a; Ruchsow et al. 2006). The ERN attenuation is explained by reduced action monitoring, which might suggest altered ACC functioning in these patients. As a result, BPD patients might not learn from errors and thus maintain their impulsive response style. In healthy individuals, smaller ERN amplitudes were associated with increased risk-taking behaviour during a card gambling task (Hewig et al. 2007) and increased risk-taking traits in adolescents (Santesso \& Segalowitz, 2009).

In the current study, we examined whether impairments of decision making are related to alterations in feedback processing in BPD. Individuals with BPD and matched healthy controls underwent a modified version of the IGT while ERPs were recorded. The FRN and feedback-related P300 were examined to elucidate the neural mechanisms of feedback processing in patients. At the behavioural level, we expected that BPD patients would show more risky decisions in the IGT and reduced learning throughout the task. Further, we assumed that higher levels of impulsivity are associated with increased risk-taking behaviour in the IGT. At the neurobiological level, we aimed at comparing ERPs following positive and negative feedback within groups. We expected diminished FRN amplitudes in BPD reflecting reduced performance monitoring. With regard to previous ERP findings, we predicted that the FRN would be related to impulsivity and risk-taking behaviour. In addition, we investigated the P300 as an indicator for the motivational significance of feedback information in BPD.

\section{Method}

\section{Participants}

Eighteen BPD patients (16 women) and 18 healthy controls (16 women) participated in this experiment. Table 1 presents the demographic and clinical measures of the study sample. All participants had normal or corrected-to-normal vision and reported no history of head trauma or neurological disease. The groups were matched with regard to age, sex and verbal intelligence, as measured with a vocabulary test (Wortschatztest; Schmidt \& Metzler, 1992). Patients were recruited from an outpatient therapy project (Berliner Borderline Versorgungsstudie, Borderline Netzwerk Berlin, Germany). Clinical diagnoses were confirmed using the Structured Clinical Interview for DSM-IV (SCID-I and SCID-II; Wittchen et al. 1997). Patients with a current or lifetime diagnosis of psychotic disorder or substance dependence were excluded. Although BPD was the primary diagnoses in all cases, 16 patients met DSM-IV criteria for one or more current co-morbid diagnoses, including anxiety disorder $(n=15)$, somatoform disorder $(n=5)$, substance abuse $(n=5)$ and eating disorder $(n=4)$. The 
Table 1. Demographic and clinical characteristics of 18 healthy controls (16 women) and 18 BPD patients (16 women)

\begin{tabular}{lcrrrrrc}
\hline & $\begin{array}{l}\text { Control } \\
\text { group } \\
(n=18)\end{array}$ & & $\begin{array}{l}\text { BPD } \\
\text { group } \\
(n=18)\end{array}$ & & $t(34)$ & $p$ \\
\hline Age, years & 27.28 & $(6.61)$ & 29.11 & $(8.06)$ & 0.75 & 0.46 \\
Verbal IQ: WST & 103.56 & $(8.55)$ & 103.56 & $(10.67)$ & 0.00 & 1.00 \\
BIS-10, total score & 68.17 & $(9.35)$ & 84.67 & $(9.03)$ & 5.39 & $<0.001$ \\
BDI, total score & - & & 21.33 & $(11.37)$ & \multicolumn{1}{c}{-} & - \\
\hline
\end{tabular}

BPD, Borderline personality disorder; IQ, intelligence quotient; WST,

Wortschatztest (German Vocabulary Test); BIS-10, Barratt Impulsivity Scale version 10 ; BDI, Beck Depression Inventory.

Data are given as mean (standard deviation).

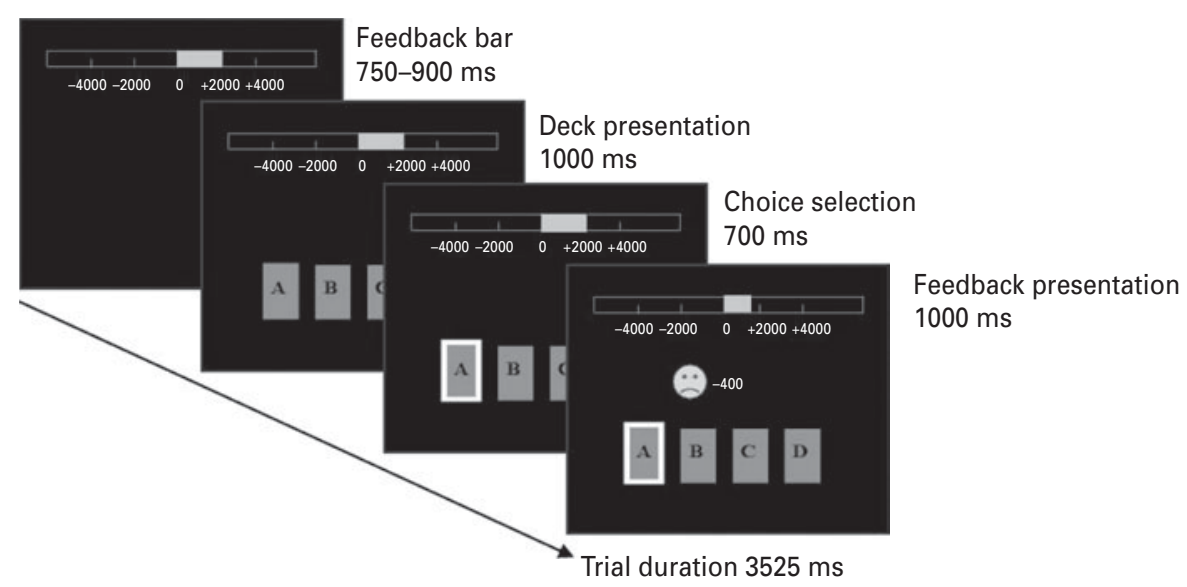

Fig. 1. Schematic depiction of the modified Iowa Gambling Task. Participants were instructed to select one card by pressing one of four response buttons corresponding to the four decks (A, B, C and D). After $700 \mathrm{~ms}$, they were shown the outcome associated with the selected card for $1000 \mathrm{~ms}$. A red frowny face together with a negative amount indicated a disadvantageous choice, while a green smiley face together with a positive amount indicated an advantageous choice. Information about the current score was indicated by a green bar, which represented a positive score or by a red bar, which represented a negative score. The experiment lasted about $40 \mathrm{~min}$.

severity of depression was assessed using the Beck Depression Inventory (BDI) within patients (Beck et al. 1961). Even though patients with current co-morbid affective disorders were excluded, 12 patients reported BDI total scores that exceeded the cut-off for clinical significance (BDI total scores $>18$ ). Further, eight patients were taking antidepressant medication at the time of testing (amitriptyline $n=1$, citalopram $n=4$, fluoxetine $n=1$, mirtazapine $n=1$, paroxetine $n=1$ ).

Healthy controls were recruited using advertisements in local newspapers. SCID-I and SCID-II interviews revealed no past or current psychiatric diagnoses. In the entire group, impulsivity was measured using the Barratt Impulsivity Scale version 10 (BIS-10; Barratt, 1985). Relative to healthy controls and in line with previous research (Rentrop et al. 2008), BPD patients described themselves as significantly more impulsive $[t(34)=5.39, p<0.001]$. Following a detailed description of the study, all participants received verbal and written explanations of the purpose and procedures of the study, and gave written informed consent. The study was approved by the local ethics committee of the Charite University Hospital, Berlin and was conducted in accordance with the Declaration of Helsinki. All participants received financial compensation ( $€ 8$ per $h$ ) for their participation.

\section{Task and procedure}

Participants underwent a computerized version of the IGT (Bechara et al. 1994) that was modified for ERP recordings (Fig. 1). Participants were presented with four decks of cards (A, B, C and D) in a horizontal line 
Table 2. Reinforcement schedule in the modified Iowa Gambling Task ${ }^{\mathrm{a}}$

\begin{tabular}{|c|c|c|c|c|c|c|}
\hline \multirow{2}{*}{$\begin{array}{l}\text { Block } \\
\text { sequence }\end{array}$} & \multicolumn{3}{|c|}{ Disadvantageous decks } & \multicolumn{3}{|c|}{ Advantageous decks } \\
\hline & A & $\mathrm{B}$ & Net loss & C & $\mathrm{D}$ & Net gain \\
\hline 1 & $+136 /-170$ & $+136 /-629$ & -170 & $+68 /-34$ & $+68 /-187$ & +170 \\
\hline 2 & $+152 /-190$ & $+152 /-703$ & -190 & $+76 /-38$ & $+76 /-209$ & +190 \\
\hline 3 & $+160 /-200$ & $+160 /-740$ & -200 & $+80 /-40$ & $+80 /-220$ & +200 \\
\hline 4 & $+176 /-220$ & $+176 /-814$ & -220 & $+88 /-44$ & $+88 /-242$ & +220 \\
\hline 5 & $+192 /-240$ & $+192 /-888$ & -240 & $+96 /-48$ & $+96 /-264$ & +240 \\
\hline 6 & $+200 /-250$ & $+200 /-925$ & -250 & $+100 /-50$ & $+100 /-275$ & +250 \\
\hline 7 & $+216 /-270$ & $+216 /-999$ & -270 & $+108 /-54$ & $+108 /-297$ & +270 \\
\hline 8 & $+232 /-290$ & $+232 /-1073$ & -290 & $+116 /-58$ & $+116 /-319$ & +290 \\
\hline 9 & $+240 /-300$ & $+240 /-1110$ & -300 & $+120 /-60$ & $+120 /-330$ & +300 \\
\hline 10 & $+256 /-320$ & $+256 /-1184$ & -320 & $+128 /-64$ & $+128 /-352$ & +320 \\
\hline 11 & $+272 /-340$ & $+272 /-1258$ & -340 & $+136 /-68$ & $+136 /-374$ & +340 \\
\hline 12 & $+280 /-350$ & $+280 /-1295$ & -350 & $+140 /-70$ & $+140 /-385$ & +350 \\
\hline $\begin{array}{l}\text { Gain-loss } \\
\text { frequency }\end{array}$ & $\begin{array}{l}50 \% \text { Gains } / 50 \% \\
\text { losses }\end{array}$ & $\begin{array}{l}80 \% \text { Gains } / 20 \% \\
\text { losses }\end{array}$ & - & $\begin{array}{l}50 \% \text { Gains } / 50 \% \\
\text { losses }\end{array}$ & $\begin{array}{l}80 \% \text { Gains } / 20 \% \\
\text { losses }\end{array}$ & - \\
\hline
\end{tabular}

\footnotetext{
${ }^{\text {a }}$ Participants completed 12 blocks with 60 trials each (720 total trials). Blocks consisted of 12 different levels of gains and losses and were presented in pseudo-randomized order. At the beginning of each block, deck positions were pseudo-randomized. Decks A and B were associated with large magnitude outcomes, while decks C and D were associated with low magnitude outcomes. Decks A and C yielded losses on $50 \%$ of the trials, while decks B and D yielded infrequent losses on $20 \%$ of the trials. In the long run, decks A and B were disadvantageous (referred to as being 'risky') as they led to a net loss over time. Decks $\mathrm{C}$ and D were advantageous (referred to as being 'safe') as they led to net gains throughout the task.
}

for $1000 \mathrm{~ms}$. Cards were selected by pressing one of four corresponding response buttons. After $700 \mathrm{~ms}$, participants were shown the outcome associated with the card selection for $1000 \mathrm{~ms}$. A red frowny face together with a negative amount indicated a disadvantageous choice, while a green smiley face together with a positive amount indicated an advantageous choice. When responses were slower than $1000 \mathrm{~ms}$, participants were instructed to respond more quickly. Each trial started with an adaptive feedback bar that informed participants about their current total score. The next trial was presented at random intertrial intervals between 650 and $950 \mathrm{~ms}$. Participants were instructed to win as much virtual money as possible, and they were told that some decks were worse than others, but that they could still win if they avoided the worst decks (Bechara et al. 1999). Decks A and B were associated with large magnitude outcomes, while decks $C$ and $D$ were associated with low magnitude outcomes. In the long run, decks A and B were disadvantageous (referred to as being 'risky') as they led to a net loss over time. Decks C and D were advantageous (referred to as being 'safe') as they led to net gains throughout the task.

Participants underwent 12 blocks of 60 trials each with short breaks between blocks (720 total trials in the modified IGT $v .100$ trials in the original IGT; Bechara et al. 1994). Table 2 presents an overview of the reinforcement schedule on each deck. Decks A and $C$ yielded losses on $50 \%$ of the trials, while decks B and D yielded losses on $20 \%$ of the trials. The lengthening was necessary to obtain a sufficient number of negative feedback trials for ERP analyses in all conditions. In order to maintain motivation throughout the experiment, each block began with 2000 points of starting credit (Bechara et al. 2000). Blocks were characterized by different levels of gains and losses and were presented in pseudo-randomized order. Furthermore, deck positions were pseudo-randomized at the beginning of each block.

\section{Electroencephalogram (EEG) recording and data analyses}

The EEG was recorded from 64 electrodes sites including $\mathrm{Cz}$ as recording reference by using an equidistant electrode system (EASYCAP $\mathrm{GmbH}$, Germany). Additional electrodes were placed below the right and left eye (IO1, IO2) to record vertical eye movements and the activity from distant muscles (neck electrode). The ground electrode was located below the left mastoid (T1). Electrode impedances were kept below $5 \mathrm{k} \Omega$. During recording, all activity was sampled digitally at a rate of $500 \mathrm{~Hz}$, using a time constant of $10 \mathrm{~s}$ and a low-pass filter of $250 \mathrm{~Hz}$. Individual electrode positions were digitized based on 
the run-time measurement of ultrasonic pulses using ELPOS (zebris Medical GmbH, Germany). Off-line, the EEG data were re-referenced to average reference and corrected for eye-movement artifacts using the multiple source eye correction method as implemented in BESA 5.1 (Brain Electrical Source Analysis; MEGIS Software $\mathrm{GmbH}$, Germany). Raw data were filtered with a low-pass filter of $40 \mathrm{~Hz}$ and a notch filter of $50 \mathrm{~Hz}$. Feedback-locked epochs were obtained for each trial, starting $200 \mathrm{~ms}$ prior to feedback onset and continuing for $1000 \mathrm{~ms}$ post-feedback. Individual averages were baseline corrected to an average activity between 200 and $0 \mathrm{~ms}$ before feedback onset. Feedback-locked epochs were excluded from further analyses if they still contained artifacts. For each participant, ERPs were averaged separately for positive and negative feedback. Grand average ERPs were filtered with a $15 \mathrm{~Hz}$ low-pass filter.

As FRN and P300 amplitudes did not always show clear peaks in individual waveforms, ERP analyses were based on mean amplitudes instead of peak amplitudes. For statistical analysis, FRN amplitudes were determined in a time window between 240 and $310 \mathrm{~ms}$ following feedback onset at electrodes Fz and FCz. The P300 was quantified at CPz and Pz and defined as the mean amplitude within 300 to $400 \mathrm{~ms}$ after feedback presentation. ERP time windows were based on the visual inspection of the grand-average waveforms and were centred around peaks (for FRN : Fz, for P300: Pz).

For behavioural analysis, deck choices were classified as advantageous (decks C and D) or disadvantageous (decks A and B). IGT net scores were calculated as the difference between the number of advantageous and disadvantageous choices (Bechara et al. 1994). IGT net scores served as the dependent variable and were compared between the groups using two-tailed $t$ tests. Due to the a priori hypotheses, IGT learning was examined by dividing the 12 blocks into terciles, each containing four blocks (blocks 1-4, 5-8, 9-12). IGT net scores were calculated for each of these terciles to identify changes in the pattern of choices (Bechara et al. 2000). Learning was then analysed by comparing the first and the final IGT terciles. To examine whether the high number of trials within the total experiment $(n=720)$ might have caused a motivational decline, all analyses were repeated for the first 60 trials (i.e. block 1 ).

ERP data were analysed by repeated-measurement analysis of variance with the between-subjects factor 'group' (BPD patients $v$. healthy controls) and the within-subject factors 'electrode' (for FRN: Fz and $\mathrm{FCz}$, for P300: $\mathrm{CPz}$ and $\mathrm{Pz}$ ) and 'feedback valence' (positive feedback $v$. negative feedback). Since the FRN amplitude was largest at the Fz electrode, correlation coefficients (Pearson's $r$ ) were used to examine

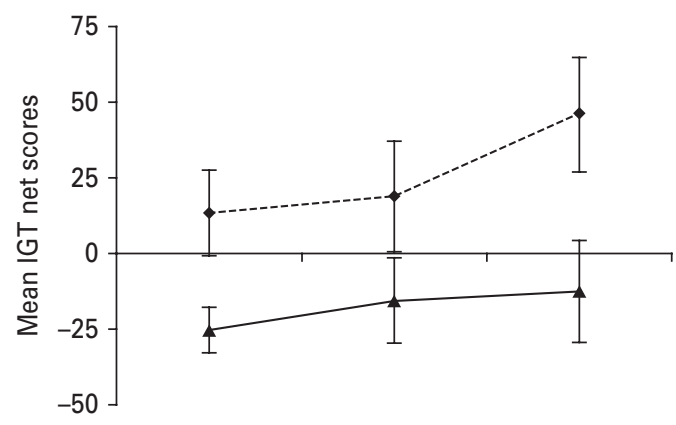

Blocks 1-4 Blocks 5-8 Blocks 9-12

Fig. 2. Iowa Gambling Task (IGT) learning for borderline personality disorder patients $(n=18,-\mathbf{\Delta}-)$ and healthy controls $(n=18,--\bullet--)$. The $\mathrm{x}$-axis represents the consecutive terciles, each containing four blocks. The y-axis represents the mean IGT net score (total number of choices from decks $C$ and D minus total number of choices from decks A and B), with standard errors represented by vertical bars.

the association between FRN magnitude at Fz and selfreported impulsivity and risky decisions in the IGT. To assess whether the FRN following negative feedback was affected by elevated levels of depressive symptoms (BDI total score), in patients additional bivariate correlations were computed within BPD patients $(n=18)$. Behavioural analyses were conducted with 'deck choices' as the within-subject factor and 'electrode' and 'feedback valence' as withinsubject factors for the ERP analyses, respectively.

\section{Results}

\section{Behavioural findings}

In BPD, deck choices resulted in a negative mean IGT net score of -53.4 (S.D. $=144.6)$, whereas healthy controls showed a positive mean IGT net score of 78.2 (S.D.=193.3). BPD patients performed significantly worse compared with healthy controls, as reflected in lower mean IGT net scores $[t(34)=2.23, p<0.05]$. Fig. 2 presents the mean IGT net scores within terciles for healthy controls and BPD patients. Across the blocks, BPD patients showed lower mean IGT net scores compared with healthy controls $(p<0.05)$. Furthermore, there was no improvement within patients $(p=0.36)$, but learning was observed in healthy controls $(p=0.05)$. To control for motivational effects, IGT performance was additionally analysed within the first 60 trials (i.e. block 1). BPD patients tended to show lower mean IGT net scores compared with controls $[t(34)=1.78, p=0.08]$. Again, there was no learning in $\operatorname{BPD}(p=0.37)$, whereas the controls tended to enhance performance throughout the first 60 trials $(p=0.09)$. 

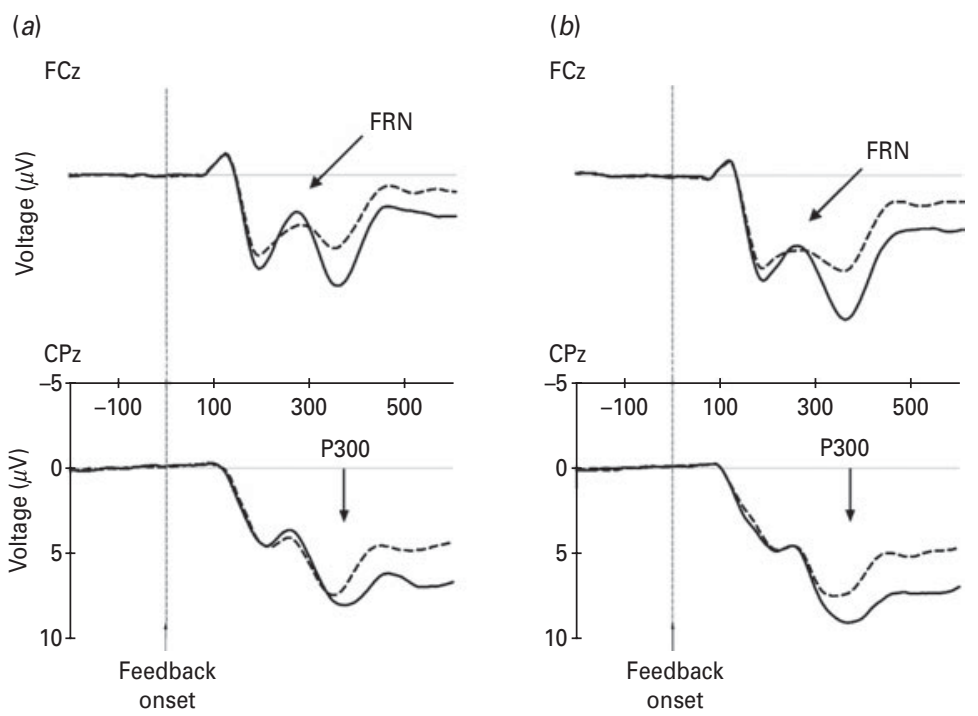

Fig. 3. Averaged feedback-locked event-related brain potential (ERP) waveforms for the feedback-related negativity (FRN) and the P300 of $(a)$ healthy controls $(n=18)$ and $(b)$ borderline personality disorder patients $(n=18)$ at Fz, FCz, CPz and Pz for positive (---) and negative (-) feedback.

\section{ERP findings}

Feedback-locked ERP waveforms following positive and negative feedback presentation are displayed in Fig. 3. With regard to the FRN, no main effect for 'feedback valence' was found $(F<1)$, but a significant interaction between 'feedback valence' and 'group' was obtained $[F(1,34)=4.16, p=0.05]$. In line with previous studies, healthy controls showed significant amplitude differences between positive and negative feedback $(p<0.02)$. In contrast, this amplitude difference was not found in patients $(p=0.60)$. Relative to healthy controls, patients had reduced (i.e. more positive) FRN amplitudes following negative feedback $(p<0.03)$ and tended to have reduced mean amplitudes following positive feedback ( $p=0.08$, Fig. $4 a)$. Additionally, a significant main effect of electrode emerged $[F(1,34)=60.26, p<0.001]$, due to larger amplitudes at Fz compared with FCz.

The P300 is also depicted in Fig. 3. A main effect for 'feedback valence' was found $[F(1,34)=31.09$, $p<0.001$, indicating that the P300 was larger following negative feedback compared with positive feedback. Importantly, there was a significant interaction between 'feedback valence' and 'group' $[F(1,34)=$ 13.65, $p<0.01]$. Fig. $4 b$ demonstrates that this interaction was caused by larger P300 amplitudes following negative feedback compared with positive feedback in the patient group $(p<0.001)$. Healthy controls, however, did not show significant P300 amplitude differences between positive and negative feedback $(p=0.18)$. For the P300, no main effect for electrode was found $(F<1)$.

\section{Correlational findings}

Across groups $(n=36)$, bivariate correlations were computed between the FRN amplitude (at Fz electrode) and impulsivity (BIS-10 total score) or mean IGT net scores. To control for the direction of correlation coefficients, mean FRN amplitudes following negative feedback were multiplied with minus 1 , so that a positive correlation indicates an increase in FRN amplitude (i.e. a more negative potential). First, a significant negative correlation between FRN amplitude and impulsivity was found $(r=-0.34, p<0.05)$, indicating that higher levels of impulsivity were associated with reduced (i.e. more positive) FRN amplitudes. Second, a significant positive correlation between FRN amplitude and IGT net score was observed $(r=0.36$, $p<0.05)$, reflecting that larger FRN amplitudes were associated with higher IGT net scores (i.e. fewer risky choices). Last, a significant negative correlation between impulsivity and IGT net score was found $(r=$ $-0.46, p<0.02)$, indicating that higher levels of impulsivity were associated with lower IGT net scores. Additionally, no significant correlation between FRN amplitude and depressive symptoms (BDI total score) was found in the patient group $(r=0.32, p=0.20)^{1} \uparrow$.

\section{Discussion}

This study focused on decision making in individuals with BPD using a modified IGT. Simultaneously, EEG recordings were assessed to clarify how feedback

\footnotetext{
$\dagger$ The note appears after the main text.
} 

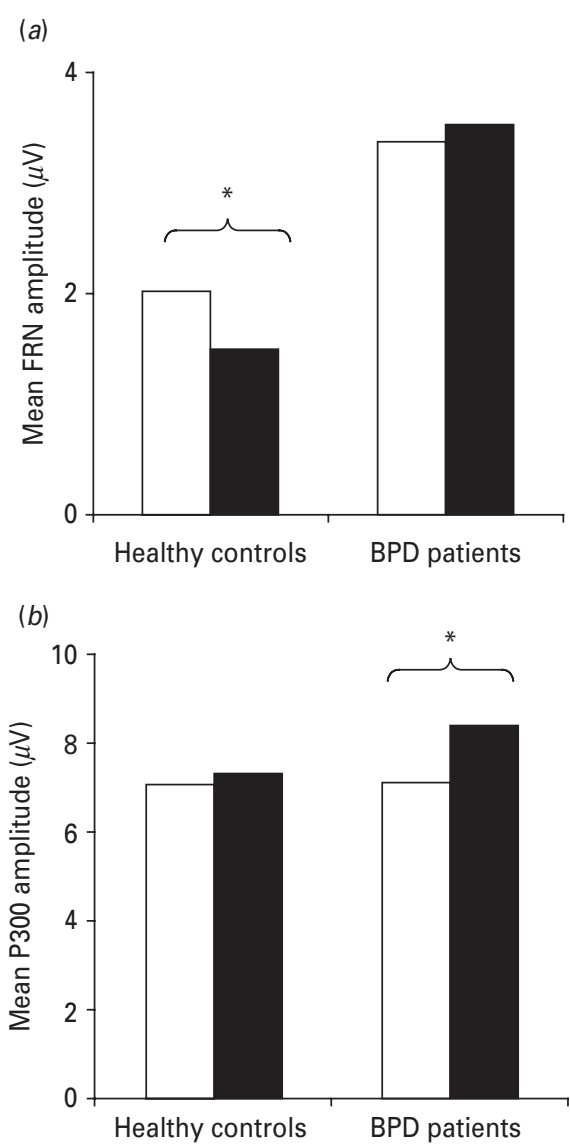

Fig. 4. Amplitudes of feedback-locked event-related brain potentials plotted as a function of group [healthy controls, $n=18$; borderline personality disorder (BPD) patients, $n=18$ ] and feedback ( $\square$, positive; $\mathbf{\square}$, negative). (a) Mean feedbackrelated negativity (FRN) amplitudes across electrodes $\mathrm{Fz}$ and FCz relative to a pre-stimulus baseline. It should be noted that the FRN is a negative-going deflection but positive $(\mu \mathrm{V})$. The larger the FRN, the less positive the deflection. (b) Mean P300 amplitudes across electrodes CPz and Pz relative to a pre-stimulus baseline. ${ }^{*}$ Mean value was significantly different from that for positive feedback $(p<0.05)$.

evaluation was related to decision making in these patients. In agreement with previous research on IGT performance, BPD patients were less likely to develop a preference for advantageous decks and preferred the risky decks (Haaland \& Landro, 2007; Maurex et al. 2009). Further, the number of risky decisions correlated with enhanced impulsivity in the entire sample. Decision-making deficits in the IGT have been found in patients with several neurological or psychiatric disorders. For instance, IGT impairment was shown in patients with $\mathrm{OFC} /$ ventromedial cortex lesions or with amygdala damage (Bechara et al. 1994, 1999). In addition, individuals with substance dependence (Bechara et al. 2001) as well as patients with impulse spectrum disorders (e.g. pathological gambling; Cavedini et al. 2002) exhibit abnormal decision making in the IGT. Consequently, decisionmaking disturbances seem to be a neurobiological hallmark of diminished impulse control and may be a trademark of impulse spectrum disorders (Hollander \& Rosen, 2000). In the present study, correlation analysis provided further evidence for an association between decision-making deficits and enhanced impulsivity. Thus, findings are in line with previous neuropsychological studies showing that diminished impulse control in BPD plays a pivotal role in decision making and planning (Bazanis et al. 2002; Lenzenweger et al. 2004).

With regard to the FRN and in agreement with previous findings (Miltner et al. 1997), healthy controls exhibited enhanced FRN amplitudes following negative feedback as opposed to positive feedback. In BPD patients, however, these FRN modulations by feedback valence were not observed. BPD patients exhibited diminished FRN amplitudes and at trend level reduced amplitudes following positive feedback, suggesting general alterations of feedback processing in BPD. The FRN is assumed to reflect ACC activity (Holroyd \& Coles, 2002), a brain region that plays a key role in feedback evaluation and learning about the consequences of actions to select more appropriate future behaviours (Ridderinkhof et al. 2004). When outcomes are worse than expected, the FRN is elicited by a phasic decrease in activity of mesencephalic dopaminergic neurons (Holroyd \& Coles, 2002; Schultz, 2002). Research on the biological basis of BPD has revealed a deficit in serotonergic activity (Silk, 2000; Skodol et al. 2002), although there is evidence that dopamine dysfunction may also be associated with BPD (Friedel, 2004). ERP results are consistent with structural as well as functional ACC alterations in BPD. Previous research has shown decreased baseline metabolism in subregions of the ACC (De La Fuente et al. 1997; Tebartz van Elst et al. 2003) as well as smaller grey matter volume in BPD (Hazlett et al. 2005). Further, studies using emotional, stressful and sensory stimuli have consistently shown deactivation of ACC in BPD (Donegan et al. 2003; Schmahl et al. 2004).

Recent ERP investigations reported an association between the magnitude of the FRN and performance adjustment and demonstrated that the FRN was more negative when participants had learned from feedback information (Frank et al. 2005). In the current study, diminished FRN amplitudes were related to deficient IGT performance and heightened impulsivity. These associations suggest that BPD patients may not learn from feedback, as reflected by the absence of developing a preference for the advantageous decks. The results of our study are in line with previous findings 
linking diminished inhibition as a possible mediating process to reinforcement learning deficiencies in BPD (Bornovalova et al. 2005). Incarcerated females with BPD (Hochhausen et al. 2002) and healthy individuals high in BPD symptoms (Chapman et al. 2008) demonstrated reduced avoidance of punishment (e.g. financial penalties).

Although there is evidence for decision-making deficits in BPD, neural correlates of these processes have not yet been investigated. ERP research has focused on error processing and has demonstrated diminished ERN amplitudes in impulsive individuals (Potts et al. 2006, but Santesso \& Segalowitz, 2009) as well as individuals with BPD (De Bruijn et al. 2006a; Ruchsow et al. 2006). Further, smaller ERNs were associated with increased risk-taking in a card gambling task (Hewig et al. 2007) and risk-taking traits in adults (Santesso \& Segalowitz, 2009). The current study expands on previous ERP findings regarding actionmonitoring alterations in BPD in that it also demonstrates deficits in feedback evaluation in these patients.

To elucidate the salience of feedback information in BPD, the P300 was investigated. The results reveal that the P300 was insensitive to feedback valence in controls, while in patients the P300 was increased following negative feedback compared with positive feedback. Previous studies also found that the P300 was insensitive to feedback valence in healthy individuals (Yeung \& Sanfey, 2004). Possibly, healthy controls already had processed feedback valence earlier, as reflected in the FRN modulation. In BPD, however, the processing of feedback valence might be delayed, and thus be reflected by the P300. Since the P300 is modulated by expectations (Hajcak et al. 2005, 2007), the increased P300 in BPD may also indicate that negative outcomes were relatively unexpected to the patients. Altered processing of negative reinforcement information in BPD is also provided by a study using a binary-outcome gamble (Kirkpatrick et al. 2007). Individuals with BPD demonstrated dysfunctional processing of loss information when the probability of gains was high. In that study, risky decision making in BPD is explained by problems using feedback information, suggesting an imbalance between the appetitive and aversive motivational states excited by available reinforcement signals. Further, ERP studies demonstrated that the P300 responded more strongly to negative feedback than to positive feedback (Frank et al. 2005) and that the amplitude increased in individuals who attributed more meaning to feedback information (De Bruijn et al. 2004). In this regard, controls may evaluate positive and negative feedback information as being equally meaningful. Perhaps, enhanced P300 amplitudes following negative feedback in BPD suggest that these patients attributed more meaning to negative feedback information. This is supported by the hypothesis that the P300 may reflect motivational processes linked to noradrenergic transmission (Nieuwenhuis et al. 2005), which play an important role in modulating the reactivity and sensitivity to environmental feedback and is considered to be associated with affective instability in BPD (Steinberg et al. 1994).

This study has some limitations. Eight patients were taking psychotropic medication at the time of testing. However, patients were only taking antidepressant medication which does not alter action-monitoring processes (De Bruijn et al. 2006b). Although patients with co-morbid affective disorders were excluded, 12 patients reported elevated symptom scores for depression as measured with the BDI (Beck et al. 1961). However, no significant correlation between FRN amplitude and depressive symptoms was found in patients. Although, decision-making deficits in BPD patients are in line with earlier studies, depressive symptoms might also have influenced IGT performance. However, previous studies employing the IGT with depressed patients have found inconclusive results, showing impaired, unaltered or superior performance (Dalgleish et al. 2004; Must et al. 2006; Smoski et al. 2008). It might be interesting to compare our findings with those based on BPD samples without any co-morbidity or depressive symptoms. But it remains questionable whether a 'pure' BPD sample would be representative considering the high comorbidity rate for BPD in general (Zanarini et al. 2004). Finally, there are possible behavioural confounds which might account for group differences. It remains unclear whether altered decision making in patients is a consequence of altered feedback processing or whether it leads to these FRN alterations. Recent empirical findings suggest that the IGT is a complex paradigm, and task complexity may also interfere with the ability to distinguish the different component processes that are implicated in task performance. IGT impairments are not simply related to reinforcement learning deficits, but may also reflect the disability to attend to, synthesize and remember complex reinforcement histories and to resolve the approachavoidance conflict that arises when a deck is associated with both reward and punishment (Fellows \& Farah, 2005; Dunn et al. 2006). Another mechanism that could explain IGT deficits is lack of motivation. Rather than being unable to make adequate decisions, impaired patient groups may simply not care enough about the negative outcomes to actively avoid them. But, in the present study enhanced P300 following negative feedback in BPD argues against motivation deficits in patients. Nonetheless, behavioural results should be interpreted cautiously since controls also 
showed some difficulties within the first block. It is possible that task modifications complicated learning also within controls and that learning occurred more implicitly compared with the original IGT.

Reduced performance monitoring is not specific to $\mathrm{BPD}$, and FRN reductions have also been reported in other groups, such as schizophrenia patients (Morris et al. 2008) and older adults (Pietschmann et al. 2008). Additional research should include adequate psychiatric control groups to determine the specific impairment of reinforcement learning in BPD. Last, it should be mentioned that the current study relies on a relatively small sample size and replication in a larger sample is needed. Notwithstanding these limitations, the present study confirms previous findings regarding altered decision making in BPD and sheds new light on cognitive impairments by combining behavioural and ERP measures.

Learning from feedback is highly important for successfully making future decisions. This study indicates that BPD patients are impaired in decision making, which might be related to a dysfunctional use of feedback information. Specifically, BPD patients did not learn to avoid disadvantageous selections, albeit they attended to negative consequences. Altered neural correlates of reinforcement learning are consistent with problems faced by individuals with BPD; namely, continued engagement in certain behaviours despite negative consequences (e.g. risky sexual behaviour, alcohol/drug use, self-harm). Impulsive decision making is a core feature of BPD, so understanding the underlying mechanisms involved in feedback evaluation could greatly have an impact on the treatment of individuals with BPD.

\section{Acknowledgements}

We thank the following people for their contributions to this work: S. Röpke, M.D. (CharitéUniversitätsmedizin Berlin, Germany); T. Pinkpank, Dipl.-Psych, R. Kniesche, Dipl.-Ing, R. Blasiak, M.A., H. Groth and S. Kessler-Scheil. We also thank J. Beyer, Dipl.-Psych., C. Steffens, Dipl.-Psych., D. Spretz, Dipl.Psych. and T. Wagner, Dipl.-Psych. of the Borderline Netzwerk Charité, Campus Benjamin Franklin, Berlin, for their assistance in patient recruitment.

\section{Declaration of Interest}

None.

\section{Notes}

${ }^{1}$ Correlations between P300 amplitude and impulsivity (BIS total score) or mean IGT net score were not significant (all $p>0.26$ ).

\section{References}

APA (2000). Diagnostic and Statistical Manual of Mental Disorders, 4th edn. American Psychiatric Association: Washington, DC.

Barratt ES (1985). Impulsiveness subtraits: arousal and information processing. In Motivation, Emotion and Personality (ed. J. T. Spence and C. E. Izard), pp. 137-146. Elsevier: North-Holland.

Bazanis E, Rogers RD, Dowson JH, Taylor P, Meux C, Staley C, Nevinson-Andrews D, Taylor C, Robbins TW, Sahakian BJ (2002). Neurocognitive deficits in decision making and planning of patients with DSM-III-R borderline personality disorder. Psychological Medicine 32, 1395-1405.

Bechara A, Damasio AR, Damasio H, Anderson SW (1994). Insensitivity to future consequences following damage to human prefrontal cortex. Cognition 50, 7-15.

Bechara A, Damasio H, Damasio AR (2000). Emotion, decision making and the orbitofrontal cortex. Cerebral Cortex 10, 295-307.

Bechara A, Damasio H, Damasio AR, Lee GP (1999). Different contributions of the human amygdala and ventromedial prefrontal cortex to decision making. Journal of Neuroscience 19, 5473-5481.

Bechara A, Dolan S, Denburg N, Hindes A, Anderson SW, Nathan PE (2001). Decision making deficits, linked to a dysfunctional ventromedial prefrontal cortex, revealed in alcohol and stimulant abusers. Neuropsychologia 39, 376-389.

Beck AT, Ward CH, Mendelson M, Mock J, Erbaugh J (1961). An inventory for measuring depression. Archives of General Psychiatry 4, 561-571.

Berlin HA, Rolls ET, Iversen SD (2005). Borderline personality disorder, impulsivity, and the orbitofrontal cortex. American Journal of Psychiatry 162, 2360-2373.

Bornovalova MA, Lejuez CW, Daughters SB, Zachary Rosenthal M, Lynch TR (2005). Impulsivity as a common process across borderline personality and substance use disorders. Clinical Psychology Review 25, 790-812.

Brown JW, Braver TS (2007). Risk prediction and aversion by anterior cingulate cortex. Cognitive, Affective and Behavioral Neuroscience 7, 266-277.

Cavedini P, Riboldi G, Keller R, D'Annucci A, Bellodi L (2002). Frontal lobe dysfunction in pathological gambling patients. Biological Psychiatry 51, 334-341.

Chapman AL, Leung DW, Lynch TR (2008). Impulsivity and emotion dysregulation in borderline personality disorder. Journal of Personality Disorders 22, 148-164.

Dalgleish T, Yiend J, Bramham J, Teasdale JD, Ogilvie AD, Malhi G, Howard R (2004). Neuropsychological processing associated with recovery from depression after stereotactic subcaudate tractotomy. American Journal of Psychiatry 161, 1913-1916.

Debener S, Ullsperger M, Siegel M, Fiehler K, von Cramon DY, Engel AK (2005). Trial-by-trial coupling of concurrent EEG and fMRI identifies the dynamics of performance monitoring. Journal of Neuroscience 25, 11730-11737. 
De Bruijn ERA, Grootens KP, Verkes RJ, Buchholz V, Hummelen JW, Hulstijn W (2006a). Neural correlates of impulsive responding in borderline personality disorder: ERP evidence for reduced action monitoring. Journal of Psychiatric Research 40, 428-437.

De Bruijn ERA, Mars RB, Hulstijn W (2004). 'It wasn't me ... or was it?' How false feedback affects performance. In Errors, Conflicts, and the Brain. Current Opinions on Performance Monitoring (ed. M. Ullsperger and M. Falkenstein), pp. 118-124. MPI of Cognitive Neuroscience: Leipzig.

De Bruijn ERA, Sabbe BGC, Hulstijn W, Ruigt GSF, Verkes RJ (2006b). Effects of antipsychotic and antidepressant drugs on action monitoring in healthy volunteers. Brain Research 1105, 122-129.

De La Fuente JM, Goldman S, Stanus E, Vizuete C, Morlan I, Bobes J, Mendlewicz J (1997). Brain glucose metabolism in borderline personality disorder. Journal of Psychiatric Research 31, 531-541.

Donegan NH, Sanislow CA, Blumberg HP, Fulbright RK, Lacadie C, Skudlarski P, Gore JC, Olson IR, McGlashan TH, Wexler BE (2003). Amygdala hyperreactivity in borderline personality disorder: implications for emotional dysregulation. Biological Psychiatry 54, 1284-1293.

Dunn BD, Dalgleish T, Lawrence AD (2006). The somatic marker hypothesis: a critical evaluation. Neuroscience and Biobehavioural Reviews 30, 239-271.

Falkenstein M, Hohnsbein J, Hoormann J, Blanke L (1990). Effects of errors in choice reaction tasks on the ERP under focused and devided attention. In Psychophysiological Brain Research (ed. C. H. M. Brunia, A. W. K. Gaillard and A. Kok), pp. 192-195. Tilburg University Press: Tilburg.

Fellows LK, Farah MJ (2005). Different underlying impairments in decision making following ventromedial and dorsolateral frontal lobe damage in humans. Cerebral Cortex 15, 58-63.

Frank MJ, Woroch BS, Curran T (2005). Error-related negativity predicts reinforcement learning and conflict biases. Neuron 47, 495-501.

Friedel RO (2004). Dopamine dysfunction in borderline personality disorder: a hypothesis.

Neuropsychopharmacology 29, 1029-1039.

Gehring WJ, Goss B, Coles MGH, Meyer DE, Donchin E (1990). The error-related negativity: an event related brain potential accompanying errors. Psychophysiology 27, 34.

Gehring WJ, Willoughby AR (2002). The medial frontal cortex and the rapid processing of monetary gains and losses. Science 295, 2279-2282.

Haaland VO, Landro NI (2007). Decision making as measured with the Iowa Gambling Task in patients with borderline personality disorder. Journal of the International Neuropsychological Society 13, 699-703.

Hajcak G, Holroyd CB, Moser JS, Simons RF (2005). Brain potentials associated with expected and unexpected good and bad outcomes. Psychophysiology 42, 161-170.

Hajcak G, Moser JS, Holroyd CB, Simons RF (2007). It's worse than you thought: the feedback negativity and violations of reward prediction in gambling tasks. Psychophysiology 44, 905-912.
Hazlett EA, New AS, Newmark R, Haznedar MM, Lo JN, Speiser LJ, Chen AD, Mitropoulou V, Minzenberg M, Siever LJ, Buchsbaum MS (2005). Reduced anterior and posterior cingulate gray matter in borderline personality disorder. Biological Psychiatry 58, 614-623.

Hewig J, Trippe R, Hecht H, Coles MG, Holroyd CB, Miltner WH (2007). Decision making in Blackjack: an electrophysiological analysis. Cerebral Cortex 17, 865-877.

Hochhausen NM, Lorenz AR, Newman JP (2002). Specifying the impulsivity of female inmates with borderline personality disorder. Journal of Abnormal Psychology 111, 495-501.

Hollander E, Rosen J (2000). Impulsivity. Journal of Psychopharmacology 14, S39-S44.

Holmes AJ, Pizzagalli DA (2010). Effects of task-relevant incentives on the electrophysiological correlates of error processing in major depressive disorder. Cognitive, Affective and Behavioral Neuroscience 10, 119-128.

Holroyd CB, Coles MG (2002). The neural basis of human error processing: reinforcement learning, dopamine, and the error-related negativity. Psychological Review 109, 679-709.

Holroyd CB, Coles MGH, Nieuwenhuis S (2002). Medial prefrontal cortex and error potentials. Science 296, 1610-1611.

Kirkpatrick T, Joyce E, Milton J, Duggan C, Tyrer P, Rogers RD (2007). Altered emotional decision making in prisoners with borderline personality disorder. Journal of Personality Disorders 21, 243-261.

LeGris J, van Reekum R (2006). The neuropsychological correlates of borderline personality disorder and suicidal behaviour. Canadian Journal of Psychiatry 51, 131-142.

Lenzenweger MF, Clarkin JF, Fertuck EA, Kernberg OF (2004). Executive neurocognitive functioning and neurobehavioral systems indicators in borderline personality disorder: a preliminary study. Journal of Personality Disorders 18, 421-438.

Links PS, Heslegrave R, van Reekum R (1999). Impulsivity: core aspect of borderline personality disorder. Journal of Personality Disorders 13, 1-9.

Maurex L, Zaboli G, Wiens S, Asberg M, Leopardi R, Ohman A (2009). Emotionally controlled decision making and a gene variant related to serotonin synthesis in women with borderline personality disorder. Scandinavian Journal of Psychology 50, 5-10.

Miltner WHR, Braun CH, Coles MGH (1997). Event-related brain potentials following incorrect feedback in a timeestimation task: evidence for a 'generic' neural system for error detection. Journal of Cognitive Neuroscience 9, 788-798.

Morris SE, Heerey EA, Gold JM, Holroyd CB (2008).

Learning-related changes in brain activity following errors and performance feedback in schizophrenia. Schizophrenia Research 99, 274-285.

Must A, Szabo Z, Bodi N, Szasz A, Janka Z, Keri S (2006). Sensitivity to reward and punishment and the prefrontal cortex in major depression. Journal of Affective Disorders 90, 209-215.

Nieuwenhuis S, Aston-Jones G, Cohen JD (2005). Decision making, the P3, and the locus coeruleus-norepinephrine system. Psychological Bulletin 131, 510-532. 
Pietschmann M, Simon K, Endrass T, Kathmann N (2008). Changes of performance monitoring with learning in older and younger adults. Psychophysiology 45, 559-568.

Polezzi D, Sartori G, Rumiati R, Vidotto G, Daum I (2009). Brain correlates of risky decision making. Neuroimage 49, 1886-1894.

Potts GF, George MR, Martin LE, Barratt ES (2006). Reduced punishment sensitivity in neural systems of behavior monitoring in impulsive individuals. Neuroscience Letters 397, 130-134.

Rentrop M, Backenstrass M, Jaentsch B, Kaiser S, Roth A, Unger J, Weisbrod M, Renneberg B (2008). Response inhibition in borderline personality disorder: performance in a Go/Nogo task. Psychopathology 41, 50-57.

Ridderinkhof KR, Ullsperger M, Crone EA, Nieuwenhuis S (2004). The role of the medial frontal cortex in cognitive control. Science 306, 443-447.

Rolls ET, Hornak J, Wade D, McGrath J (1994). Emotionrelated learning in patients with social and emotional changes associated with frontal lobe damage. Journal of Neurology, Neurosurgery and Psychiatry 57, 1518-1524.

Ruchsow M, Walter H, Buchheim A, Martius P, Spitzer M, Kachele H, Gron G, Kiefer M (2006). Electrophysiological correlates of error processing in borderline personality disorder. Biological Psychology 72, 133-140.

Ruocco AC (2005). The neuropsychology of borderline personality disorder: a meta-analysis and review. Psychiatry Research 137, 191-202.

Santesso DL, Segalowitz SJ (2009). The error-related negativity is related to risk taking and empathy in young men. Psychophysiology 46, 143-152.

Sato A, Yasuda A, Ohira H, Miyawaki K, Nishikawa M, Kumano H, Kuboki T (2005). Effects of value and reward magnitude on feedback negativity and P300. Neuroreport 16, 407-411.

Schmahl CG, Vermetten E, Elzinga BM, Bremner JD (2004). A PET study of memories of childhood abuse in borderline personality disorder. Biological Psychiatry 55, 759-765.
Schmidt K-H, Metzler P (1992). Wortschatztest (WST). Beltz Test GmbH: Weinheim.

Schultz W (2002). Getting formal with dopamine and reward. Neuron 36, 241-263.

Silk KR (2000). Borderline personality disorder. Overview of biologic factors. Psychiatric Clinics of North America 23, 61-75.

Skodol AE, Siever LJ, Livesley WJ, Gunderson JG, Pfohl B, Widiger TA (2002). The borderline diagnosis II: biology, genetics, and clinical course. Biological Psychiatry 51, 951-963.

Smoski MJ, Lynch TR, Rosenthal MZ, Cheavens JS, Chapman AL, Krishnan RR (2008). Decision making and risk aversion among depressive adults. Journal of Behavior Therapy and Experimental Psychiatry 39, 567-576.

Steinberg BJ, Trestman RL, Siever LJ (1994). The cholinergic and noradrenergic neurotransmitter systems and affective instability in borderline personality disorder. In Biological and Neurobehavioral Studies of Borderline Personality Disorder (ed. K. R. Silk), pp. 41-62. American Psychiatric Press: Washington, DC.

Tebartz van Elst L, Hesslinger B, Thiel T, Geiger E, Haegele K, Lemieux L, Lieb K, Bohus M, Hennig J, Ebert D (2003). Frontolimbic brain abnormalities in patients with borderline personality disorder: a volumetric magnetic resonance imaging study. Biological Psychiatry 54, 163-171.

Wittchen H-U, Zaudig M, Fydrich T (1997). SKID. Strukturiertes Klinisches Interview fuer DSM-IV. Achse I und II. Handanweisung. Hogrefe: Gottingen.

Yeung N, Sanfey AG (2004). Independent coding of reward magnitude and valence in the human brain. Journal of Neuroscience 24, 6258-6264.

Zanarini MC, Frankenburg FR, Hennen J, Reich DB, Silk KR (2004). Axis I comorbidity in patients with borderline personality disorder: 6-year follow-up and prediction of time to remission. American Journal of Psychiatry 161, 2108-2114. 